\title{
Research Progress on Degradation of PPCPs by Micro-Algae
}

\author{
Peng Xie ${ }^{1}$, Chuan Chen ${ }^{1}$, Shih-Hsin Ho ${ }^{1}$, Jing Peng ${ }^{1,2}$, Nanqi Ren ${ }^{1,}$ * \\ ${ }^{1}$ School of Municipal and Environmental Engineering, Harbin Institute of Technology, Harbin, China \\ ${ }^{2}$ The Architectural Design and Research Institute of HIT, Harbin Institute of Technology, Harbin, China
}

\section{Email address:}

Sasori_XP@163.com (Peng Xie), echo110244@126.com (Chuan Chen), stephen6949@msn.com (Shih-Hsin Ho), bepa2000@163.com (Jing Peng), rnq@hit.edu.cn (Nanqi Ren)

*Corresponding author

\section{To cite this article:}

Peng Xie, Chuan Chen, Shih-Hsin Ho, Jing Peng, Nanqi Ren. Research Progress on Degradation of PPCPs by Micro-Algae. Science Discovery. Vol. 5, No. 3, 2017, pp. 192-198. doi: 10.11648/j.sd.20170503.16

Received: April 3, 2017; Accepted: May 4, 2017; Published: May 11, 2017

\begin{abstract}
Pharmaceuticals and personal care products (PPCPs) is currently one of the most concerned new pollutions. PPCPs is a very large system, including antibiotics, anti-inflammatory drugs, lipid lowering drugs, musk and hormones, etc. Up to now, more than 3000 kinds of drugs have been developed, and the annual output of raw materials reaches $2 \times 10^{6}$ tons; similarly, known PPCPs also reached thousands of species, and raw materials annual output reaches $1 \times 10^{6}$ tons annually. In this paper the source and emission of PPCPs in municipal wastewater are summarized as well as an overview of traditional reduction theory, technology and advanced treatment process of PPCPs, Furthermore, the paper highlights the research results and progress of new technology for PPCPs removal by algae and aquatic algae, which provides a new idea for the removal of PPCPs. Finally, the paper proposes an ongoing research of algae-based PPCPs removal process in the future.
\end{abstract}

Keywords: Pharmaceuticals and Personal Care Products (PPCPs), Biodegradation, Microalgae

\section{利用微藻降解PPCPs的研究进展}

谢鹏 $^{1}$, 陈川 ${ }^{1}$, 贺诗欣 ${ }^{1}$, 彭晶 ${ }^{1,2}$, 任南琪 ${ }^{1 *}$

${ }^{1}$ 市政环境工程学院, 哈尔滨工业大学, 哈尔滨市, 中国

${ }^{2}$ 建筑设计研究院, 哈尔滨工业大学, 哈尔滨市, 中国

邮箱

Sasori_XP@163.com（谢鹏），echo110244@126.com（陈川），stephen6949@msn.com（贺诗欣），bepa2000@163.com（彭晶）， rnq@it. edu.cn（任南琪）

摘要: 药品和个人护理品 (PPCPs种类繁多, 包括抗生素、消炎药、降脂药、鹿香和激素等。药品已知化合物种类超过 3000 多种, 原料年产量达 $2 \times 10^{6}$ 吨; 个人护理品已知化合物种类也达到上千种，原料年产量达 $1 \times 10^{6}$ 吨。本文归纳了城 市污水中PPCPs的来源及排放情况。在概述PPCPs的传统减量化技术理论和深度处理工艺的基础上，重点介绍了利用微 藻和水生藻类植物去除PPCPs的新型技术的成果和研究进展, 为去除PPCPs提供了一种新的思路。提出未来从藻类新型 技术降解机理及PPCPs的转化规律的角度进一步开展的研究趋势。

关键词: 药品和个人护理品, 生物降解, 微藻 


\section{1. 引言}

近年来, 随着污水生物处理技术研究的进一步深入, 研究对象由原来的典型的污染物, 如含氮、硫化合物, 多 环芳烃类化合物、二噁英及农药逐渐过渡到 “新兴污染 物”上。所谓的 “新兴污染物”一般指微污染物（Micro contaminant), 其中包括药品与个人护理品、内分泌干扰 物 (Endocrine disrupting compounds, EDC)、持久性有 机污染物 (Persistent Organic Pollutants, POPs) 和 微塑料 (Micro-plastic) 等 [1-3]。其中PPCPs所含的化学 物质种类最多, PPCPs 具有较强的生物活性、强极性、生 物富集性和化学持久性, 基本是以痕量浓度存在于环境 中。人类医药、化妆品、兽类医药以及农药的生产和使用 是其在环境中产生的主要途径 [4]。

这些目标污染物进入到自然水体中开始逐级富集, 使 原本微量的污染物浓度成倍提升, 导致自然水体中的微生 物及动植物体内产生耐药性。部分PPCPs在自然界中很难 降解, 已有报道称, PPCPs最后会随着食物链进入到人体 中, 增加人体致癌和致内分泌紊乱性的风险 [5]。随着新 的药品逐渐被开发和人们的消费水平状况在日益提升, 经 济越发达的城市所产出的PPCPs越多。关于如何去除PPCPs 就成为了重点研究对象。PPCPs的去除方式除了传统生物 处理和深度处理, 最新研究表明可利用藻类去除 PPCPs [6]。

\section{PPCPs的排放及分布情况}

第一例关于PPCPs的法规条例源于上个世纪70年代, 是由美国药品与食品管理局推行颁布的人与兽药新药品 授权相关的环境风险评价制度。随后欧盟、澳大利亚、加 拿大及日本的相关管理机构先后推出关于涉及环境微污 染物药品的使用及处理的相关制度，较著名的有欧盟 “海 神计划” 和美国食品和药物管理局颁布的 “联合国食品、 药品和化妆品法”。在亚洲, 日本已经参与VICH的文件编 写并且已经完成了I和II阶段的计划实施 [7]。目前, 目前, 中国还没有相关的法律法规的制定，对于各类的微污染物 的排放也没有明确的标准。
通常在城市, 家庭、医院、工业产生的PPCPs主要通 过城市污水进行排放, PPCPs 在污水处理厂出水中的浓度 在 $n g \cdot \mathrm{L}^{-1}$ 到 $\mu \mathrm{g} \cdot \mathrm{L}^{-1}$ 级之间。在农村, PPCPs主要通过径流与 地下渗流的形式流入至环境中。PPCPs在地表水中浓度范 围一般在 10 到 $1000 \mathrm{ng} \cdot \mathrm{L}^{-1}$, 在地下水中浓度一般在 $\mu \mathrm{g} \cdot \mathrm{L}^{-1}$ 水平左右 $[8]$ 。目前, 在城市污水厂出水检测中仍存在大 量的药品和个人护理品 [3]。相对环境中的微污染物的来 源, 城市污水排放是PPCPs进入到环境中的主要途径。

在集中式污水处理厂初始设计中, 其目标在于进一步 除氨和脱氮除磷, 没有用来处理PPCPs此类污染物。因此 对于微污染物中大多数极性和持久性化合物只能被小部 分去除, 在某些情况下对于部分PPCPs去除没有任何效果。 多种不同浓度的PPCPs在自然水体中富集后, 在相对较低 的浓度也可以发挥毒理效应。为此, 去除PPCPs方法开始 被研究。由于PPCPs的种类繁多, 本文总结了目前环境中 几类常见的PPCPs (见表1)。

抗生素（包括人类药品和兽药）是PPCPs中含量和种 类最多, 应用最广泛的一类重要目标污染物, 可以使人体 产生抗药性，在饮用水和污水处理厂排水中被列为检测项 目。抗生素随着生活污水和工业废水的排放, 源源不断地 输入到生态系统中, 其在自然水体中的传播和保持细菌病 原抗体性方面存在潜在效应。此外, 污水中的抗生素浓度 随季节的变化也有所不同。对污水处理厂出水以及环境地 表水中的抗生素进行了调查, 发现了磺胺类药物、大环内 酯物、青霉素和四环素类等抗生素类物质, 它们的浓度在 废水和自然水体中基本在 $\mu \mathrm{g} \cdot \mathrm{L}^{-1}$ 浓度范围 [9-12]。随后, 在欧美和北美等地区有关于污水处理厂、地表水及地下水 中的抗生素的存在浓度和降解途径开始进行了研究。 Sacher等 [13] 检测德国Baden-Württemberg地下水井的抗 生素时, 发现至少存在3处地下水含有红霉素和磺胺甲恶 唑（由八种化合物所组成的抗生素）。通常通过一个河流 水域中抗生素浓度可以判断出该地区的抗生素消费状况。 2002年, Golet等[14]在瑞士的Glatt河流调查发现当地主 要使用的抗生素有氟喹诺酮类、环丙沙星和氟哌酸, 并测 得出水的质量浓度为36 106 $\mu \mathrm{g} \cdot \mathrm{L}^{-1}$ 。

表1 环境和实验研究中一些典型的PPCPS。

Table 1. Some typical PPCPS in environmental and experiment.

\begin{tabular}{|c|c|}
\hline 种类 & 名称 \\
\hline 消炎药 & $\begin{array}{l}\text { 阿司匹林、扑热息痛、萗普酮、奥沙普秦、异丙阿托品、曲马朵、丙氧芬、菜普生、布洛芬、水杨酸、醋氨酚、双氯芬 } \\
\text { 酸、吲哚美辛、狮甲新甲羊萗丙酸（EU）、异丙安替比林 }\end{array}$ \\
\hline 抗生素 & 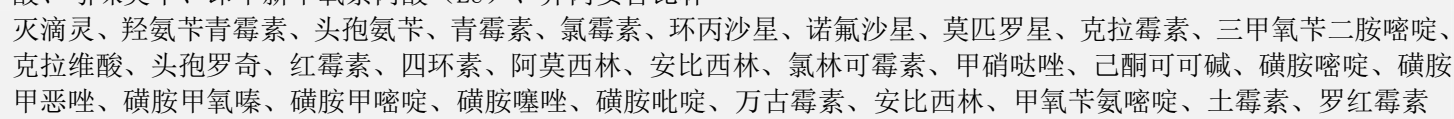 \\
\hline 血脂调节药 & 阿托伐他汀、辛伐他汀、苯扎贝特、冠心平, 吉非罗平、吉非贝齐（诺衡）、氯贝酸、苯氧戊酸、必降脂 \\
\hline 抗糖尿病药物 & 甲氟明二甲双胍、曲格列酮 \\
\hline 抗癫㾁药物 & 卡马西平、2-丙基戊酸钠、加巴喷丁、苯妥英 \\
\hline$\beta$-阻抗剂 & 美托洛尔、阿替洛尔、心安得 \\
\hline 抗阻胺剂 & 雷尼替丁、贾氰咪胍 \\
\hline 阻钙剂 & 戊脉安、地尔硫卓 \\
\hline 精神调理药物 & 威博丁、舍曲林、奈法唑酮、安定、氟西汀 \\
\hline 肌肉弛缓药 & 肌安宁 \\
\hline 利尿药 & 速尿灵、双氢克尿塞 \\
\hline
\end{tabular}




\begin{tabular}{|c|c|}
\hline 种类 & 名称 \\
\hline 拟交感（神经） & 舒喘宁 \\
\hline 解充血药 & 伪麻黄碱 \\
\hline 抗痛风剂 & 别嘌呤醇 \\
\hline 造影剂 & 碘帕醇、碘普罗胺、泛影酸钠、碘美普尔、复方泛影葡胺、碘海醇 \\
\hline 抑制细胞药物 & 安道生、异磷酰胺 \\
\hline 杀菌消毒剂 & 三氯生、联磺甲氧苄啶、联二苯、氟甲喹 \\
\hline 激素类 & $17 \mathrm{a}$-乙炔雌二醇、睪丸激素、己炔雌酚、 $17 \mathrm{\beta}$-雌二醇、双酚 $\mathrm{A}$ \\
\hline 降血压药 & 美多心安 \\
\hline 鹿香 &  \\
\hline 其他 & 咖啡因、异环磷酸钠、萘心安、辛基苯酚 \\
\hline
\end{tabular}

\section{3. 传统去除PPCPs的研究}

\section{1. 传统污水处理工艺去除PPCPs}

基于传统生物污水处理技术建立活性污泥法去除 PPCPs 的基础理论, 其主要过程分三个部分: 污泥吸附、 空气吹脱和生物转化。其中污泥吸附是主要去除途径, 污 泥吸附一般发生在初沉池和二沉池内, 基于微污染物与污 泥分离的机理可以分为两种主要的机理: 吸收和吸附。吸 收一般指活性污泥具有亲脂性的细胞膜与污染物中部分 脂肪族或芳香族发生了疏水作用; 而吸附是指污染物的带 正电荷的基团与微生物带负电荷的基团产生了表面静电 作用。如Ternes等 [15]发现卡马西平、氯贝酸、安定和双 氯芬酸等药品在初沉池和二沉池内的污泥吸附平衡常数 不同, 某些PPCPs 在二沉池的吸附常数要比初沉池的大 10 倍甚至 10 倍以上。在一级处理系统中添加混凝剂, 可以达 到较高的去除效果, Carballa等 [16]指出物化处理对于亲 脂性、中性和偏酸条件下的水体中PPCPs去除效率可达 $20 \%$ 70\%左右。因此, 一级处理工艺若采用混凝一絮凝的工 艺, 二级处理将无需深度处理便可以达到很好的去除 PPCPs 效果 [17]。在实际中, 污泥吸附去除效果较好, 但 不能从污泥中彻底降解这些目标污染物, PPCPs会随着时 间在污泥中进行富集。一旦达到饱和吸附浓度, 就会随出 水进入环境, 没有在真正意义上将PPCPs 从环境中去除。

气提法是利用水蒸气作为载气, 通过污水与水蒸气的 接触, 将一些有挥发性微污染物按一定比例扩散到气相 中, 此法可以用于去除一些易挥发或水溶性较差的物质, 如个人护理品中的硅氧烷具有高挥发性, 近 $90 \%$ 的环状挥 发性甲基硅氧烷 (cVMS) 会挥发到大气中, 从而在污水中 得到去除 [18]。同样通过汽提法可去除的微污染物还包括 壬基酚、二噁英和包含穈香类的个人护理品等。汽提法并 没有真正意义上的降解微污染物, 而是将微污染物以一种 方式从水相向气相中进行转移, 而往往大部分挥发性的 PPCPs 对人体有毒。因此, 在实际工程中需要安装气体回 收装置, 以减轻PPCPs对环境的危害作用。

生物转化被认为是降解PPCPs 的重要途径之一, Schwarzenbach等 [19]建议采用活性污泥生物转化工艺去 除PPCPs, 研究表明污泥龄和厌氧条件是决定PPCPs去除效 果的关键因素。Clara等 [20]发现在污水处理厂中, 当污 泥龄小于等于 4 天时, 没有发现PPCPs被生物降解的痕迹; Göbe1等 [21] 发现当污泥龄大于 15 天时, 可以观察到部分 PPCPs（如壬基酚、红霉素、克拉霉素等）去除效果明显;
在庆氧条件下, 许多的PPCPs去除效果更好, 其中抗生素 的去除率最高, 可达到 $80 \%$ 以上; 菜普生的在厌氧中温的 条件下去除率在 $85 \%$ 左右, 若在高温条件下可达到 $90 \%$ 左 右。此外, 通过污泥厌氧消化反应, 磺胺类抗生素和罗红 霉素的去除率高于 $80 \%$, 激素类药物如 17 B - 雌二醇的去除 率为60\% 80\%[22]。传统生物处理技术对PPCPs减量化起到 了一定的效果, 但传统生物处理技术并不是对所有PPCPs 降解都有效。有些PPCPs在庈氧污泥降解过程中起到促进 作用 $[23,24]$, 但部分PPCPs如卡马西平也会起到抑制作用 [25-27], 具体降解机理还需深入研究探讨。目前关于 PPCPs的生物降解途径和降解产物的研究较少。对生物降 解PPCPs产生的副产物毒理学研究还有待继续深入。

\section{2. 高级处理工艺}

除上述传统的生物处理法外, 还可以通过高级处理技 术对PPCPs进行降解，通常高级处理技术包括臭氧处理、 高级氧化处理（AOP）、膜过滤、活性炭吸附 [28]。目前, 在德国Baden-Württemberg在2020年底前将所有城市污水 处理厂都建造并配置以臭氧氧化、高级氧化、膜、活性炭 和生物降解为主的工艺作为四级处理装置。除此之外, 瑞 士筹划在 2031年前将全国50\%的厂都进行配置四级污水处 理工艺的改造。设置四级处理工艺的主要目的是将经过生 物处理和化学处理工艺还在废水中残留的微污染有机物 质进行有效的去除。目前已有研究表明, 引入四级深度处 理工艺可以处理可降解药品、激素和农药, 并且可以将污 水中的微塑料物质分离去除 [29]。

臭氧处理是将臭氧通过注射或者扩散输入的方式进 入液相来去除目标污染物的高级工艺。臭氧与水发生反应 生成羟基自由基, 羟基自由基在水中是强氧化剂, 会选择 性的与活性芳香烃官能团、共轭双键和非质子化胺进行反 应; 在一般情况下, 电子基团增强的臭氧氧化, 而吸电子 基团降低反应速率。Von Gunten等 [30]发现双氯芬酸、 $17 a$-乙炔雌二醇、罗红霉素和氨甲酰氮草可以与臭氧发 生反应产生多种氧化物, 从而得到降解; Huber等 [31]利 用臭氧去除河水中的苯扎贝特、卡马西平、地西泮、布洛 芬和碘普罗胺等污染物时, 发现除了苯扎贝特的去除率较 低以外, 其他目标污染物的去除率均在 $97 \%$ 以上。目前, 在经济合理的前提条件下, 对二级处理工艺后加臭氧处理 装置可减少环境水体对PPCPs的负荷。但有些臭氧氧化生 成的氧化产物或副产物具有毒性, 在采用臭氧氧化处理技 术之前, 需对反应机理及处理水质进行毒理学评估, 以免 对生态环境产生毒性危害 [32]。 
高级氧化处理工艺在处理过程中形成活性较高的羟 基, 并与其他物质形成氧化产物从而去除目标污染物。常 见的高级氧化处理方法有 $\mathrm{O}_{3} / \mathrm{H}_{2} \mathrm{O}_{2} 、 \mathrm{H}_{2} \mathrm{O}_{2} / \mathrm{UV}$ 和Fenton法等。 $\mathrm{O}_{3} / \mathrm{H}_{2} \mathrm{O}_{2}$ 法是在臭氧氧化装置上进行改进而实现的。 I jeplaar等 [33] 在 2000 年最早发现可以通过 $0_{3} / \mathrm{H}_{2} \mathrm{O}_{2}$ 法去 除PPCPs, 农药类阿特拉津去除率可达到 $99 \%$, 苯嫝类除草 剂的去除率可达到 $80 \%$ 左右; Andreozzi [34, 35] 通过 $\mathrm{H}_{2} \mathrm{O}_{2} / \mathrm{UV}$ 法去除扑热息痛和氯贝酸, 在 17 W低压紫外灯的照 射下, 在投加 5 和 $20 \mathrm{mmol} \cdot \mathrm{L}^{-1}$ 的 $\mathrm{H}_{2} \mathrm{O}_{2}$ 条件下, 扑热息痛的去 除率在 $21 \% \sim 40 \%$ 之间; 在投加 $1.0 \mathrm{~mol} \cdot \mathrm{L}^{-1}$ 的 $\mathrm{H}_{2} \mathrm{O}_{2}$ 时, 氯贝酸 被完全去除。Fenton法在中性pH条件下, 不易生成羟基, 所以对PPCPs的去除率较低 [36]。总体看来高级氧化工艺 处理效果好, 也较为经济。但是会对环境中释放多种氧化 产物及副产物, 与臭氧氧化产物相比毒性更高, 所以高级 氧化不宜投入到实际工程中使用。

此外降解PPCPs的深度处理方法还有膜过滤和活性炭 吸附。膜过滤一般采用超滤法和反渗透法 [37], 能将大部 分PPCPs截流, 但水中或副产物中的二价离子容易在膜上 形成沉淀, 影响过滤; 其工艺成本也非常昂贵, 不宜应用 于实际污水处理中。活性炭吸附去除PPCPs效率高, 能在 源头对污染物进行截流, 并有效地阻断降解副产物对环境 的输出 $[38,39]$ 。但在处理成本上需要理论证据支持。

\section{4. 基于藻类降解PPCPs的新技术研究}

自上世纪50年代以来, 利用微藻污水处理系统去除营 养物、重金属、细菌和蠕虫卵的研究已经进行开展, 研究 表明藻类在处理普通生活污水时回收营养能力较高, 可以 将生活污水中的氮和磷当做营养物质吸收到细胞中 [40], 使污水中氮磷浓度变低, 同时通过这种异养的方式提高藻 类产量。当水中存在氨和硝酸盐时, 大量繁殖的微藻也可 以作为一种新型的生物能源和肥料生产源 [41-44]。除了上 述传统的PPCPs去除技术外，为了寻找一种简便、经济、无 害化的生物降解工艺用来去除PPCPs, 利用微藻处理PPCPs 的理论被提出。但在当时没有关于利用微藻去除PPCPs的深 入研究, 因此藻类在废水中PPCPs处理的应用非常有前景。

\section{1. 微藻对于PPCPs去除的研究}

利用藻类去除PPCPs的早期研究起源于上个世纪 80 年 代。当时对地表水中的PPCPs种类进行调查, 在发现含有 多种微量抗生素类、农药类等污染物后, 主要针对PPCPs 对自然水体中的浮游生物、甲壳类动物（如虾类、蟹类） 的毒性效应做了一定程度的研究, Harrass等 [45]在 1985 年利用链霉素对小球藻、栅藻、丝藻和衣藻进行毒理学实 验, 结果发现当链霉素浓度小于 $21 \mathrm{mg} \cdot \mathrm{L}^{-1}$ 时, 小球藻、栅 藻、丝藻生长比较旺盛, 但是衣藻在链霉素浓度为 $0.66 \mathrm{mg} \cdot \mathrm{L}^{-1}$ 就已经被抑制生长了。所以微污染物浓度的差 异对于不同种属的藻类生长影响是不同的, 有些微藻甚至 可以利用目标污染物促进生长。

Brittan A. Wi1son等 [46]对堪萨斯州奥西拉污水处 理厂进水与出水进行实时采样, 并研究了水中环丙沙星、 三氯生和壬基酚聚氧乙烯醚三种PPCPs对藻类的生长速率
的影响。静态实验中, 三氯生, 环丙沙星和壬基酚聚氧乙 烯醚投加量分别 $0.12 \mu \mathrm{g} \cdot \mathrm{L}^{-1} 、 0.15 \mu \mathrm{g} \cdot \mathrm{L}^{-1} 、 0.2 \mu \mathrm{g} \cdot \mathrm{L}^{-1}$ 。在 测藻类生长曲线时, 先将混合液经过玻璃纤维过滤器, 然 后将过滤后得到的藻类放在 $-20^{\circ} \mathrm{C}$ 的环境里冷藏, 之后将 $90 \%$ 的乙醇煮沸。在乙醇中提取叶绿素 $\mathrm{a}$ 的浓度, 最后用苂 光光度计进行测量。结果表明, 三种药品对于指数生长期 的藻类没有显著的影响, 但在最终的生物量产量中有明显 的差异。其中, 壬基酚对藻类抑制生长影响最大。环丙沙 星主要影响蓝藻和绿藻的生长, 三氯生主要影响衣藻和栅 藻的生长。Brittan A. Wilson认为三种PPCPs 可能对藻类 的结构和群落功能存在着潜在的影响, 这种现象在自然环 境中的持续存在可能导致水生生物营养处理能力和食物 网结构发生变化。

PPCPs会随着地表水最终进入海洋, 对海洋生态环境 产生威胁。随着研究的深入, 藻类的选取也从淡水藻延伸 到海水藻。DeLorenzo等 [47]分析了海水检测中常见的六 种PPCPs 辛伐他汀、氯贝酸、双氯芬酸、卡马西平、氟西 汀和三氯生对杜氏盐藻的毒性效应。发现三氯生影响藻类 养分循环和营养供应源效果比较明显, 从而使藻类种群大 大减少。研究还表明, 如果多种PPCPs混合存在于水体中, 那么对藻类的毒性可能会低于单个药物对藻类的毒性水 平。但这些混合PPCPs对水生生物的毒性影响是目前面临 重要的环境问题。

近年来, 关于PPCPs对于环境中微藻的毒理学效应的 研究渐转变为利用微藻降解PPCPs。Víctor Matamoros等 [48] 利用不同水质、光辐射时间, 是否曝气等条件, 研究 了小球藻和栅藻对咖啡因、布洛芬、佳乐廊香、磷酸三丁 酯、4-辛基酚、丁氧基磷酸三乙酯和卡马西平的降解趋势。 结果表明, 咖啡因与布洛芬的去除效果非常明显, 其中咖 啡因的微藻去除率比活性污泥降解去除率提高 $40 \%$ 。这两 种污染物的去除途径主要是微藻降解而并非被吸附。对于 微藻去除丁氧基磷酸三乙酯和卡马西平的效果不好, 其降 解的途径主要是微藻吸附。经过进一步的研究发现单细胞 的微藻不吸收卡马西平。Kang Jian等 [49]利用小球藻对 人体尿液中双氯芬酸, 布洛芬, 对乙酰氨基酚、美托洛尔、 卡马西平、甲氧苄氨嘧啶等六种PPCPs的降解进行研究, 发现通过小球藻吸附去除的PPCPs 不到总去除率的 $20 \%$ 。通 过生物降解和光降解可以使 60 100\%的双氯芬酸, 布洛芬, 对乙酰氨基酚和美托洛尔被去除。其中卡马西平和甲氧苄 氨嘧啶的去除率分别不超过 $30 \%$ 和 $60 \%$ 。目前环境中存在的 这六种目标污染物浓度不会抑制小球藻的生长。此外, 藻 类处理系统在去除污染物的同时还可从源废水中分离并 回收营养物质供自身应用, 营养丰富的藻类可以收集并运 用在农业上和工业上, 例如利用藻类作肥料, 富含蛋白质 的饲料或生物燃料。

微藻类废水处理工艺如高效藻类塘（HRAPs）在世界 上也越来越受到关注。高效藻类塘是由微藻与细菌共同组 成的。在这样的藻菌共生系统中, 由异养菌降解产生有机 质提供藻类生长, 微藻进行光合作用提供氧气供好氧菌存 活; 目前, 传统活性污泥法去除PPCPs已被广泛研究, 但 是利用HRAPs 去除PPCPs的完整工艺技术尚未被开发出来。 Ignacio de Godos等 [50]确定在HRAPs合成废水处理中四 
环素的去除机制, 在HRAPs模拟反应器中先添加合成废水, 46 天之后, 加入小球藻和 $2 \mathrm{mg} \cdot \mathrm{L}^{-1}$ 的四环素。到第 62 天, 四 环素去除率稳定在 $69 \pm 1 \%$, 依据批量检测表明, 四环素主 要的去除途径是通过光降解和吸附。在实验期间藻类对化 学需氧量 (COD) 去除没有明显的效果。Víctor Matamoros 等 $[51]$ 设计了一个表面积为 $1.5 \mathrm{~m}^{3}$ 、体积为 $0.5 \mathrm{~m}^{3}$, 并配有 摚拌桨轮的调节池来模拟高效藻类塘。调节池中投加混合 藻类, 通过设置水力停留时间 (HRT) 和温度, 对生活污 水中PPCPs的浓度进行实时监测。去除率如表2所示。

表2 高效藻类塘对生活污水中一些典型PPCPs的去除效果。

Table 2. Removal efficiency of some typical PPCPS from domestic sewage by HRAPS.

\begin{tabular}{|c|c|}
\hline 去除率 & PPCPs种类 \\
\hline$>90 \%$ & $\begin{array}{l}\text { 咖啡因, 对乙酰氨基酚, 布洛芬, 二氢茉莉酮酸甲酯、 } \\
\text { 氢化肉桂酸 }\end{array}$ \\
\hline $60 \% \sim 90 \%$ & $\begin{array}{l}\text { 氧苯酮, 酮洛芬, 5-甲基-苯并三氮唑、䒺普生、佳 } \\
\text { 乐麇香, 三磷酸盐、三氯生, 双酚A、辛基酚 }\end{array}$ \\
\hline $40 \% \sim 60 \%$ & $\begin{array}{l}\text { 双氯芬酸钠、苯并三氮唑, 苯酯、开司米酮、二嗪磷、 } \\
\text { 苯并噻唑、 } 2,4-D 、 \text { 阿特拉津 }\end{array}$ \\
\hline$<40 \%$ & 卡马西平、尼泊金甲酯、三（2-氯乙基）磷酸酯 \\
\hline
\end{tabular}

由表2可见, 微类比传统活性工艺对部分PPCPs 去除率 高, 例如布洛芬在微藻系统中去除率大于 $90 \%$, 而在厌氧 消化中去除率为 $45 \pm 15 \%$ 。

\section{2. 水生藻类植物对PPCPs去除的研究}

除了微藻可以降解PPCPs, 一些水生藻类植物也可以 对PPCPs进行降解。Daughton等 [52]发现PPCPs在进入环境 中时导致水生植物与浮游生物的耐药性增强, 并使微生物 群落发生了变化。Víctor Matamoros等 [53]利用金鱼藻、 伊乐藻和浮萍等水生植物对双氯芬酸、布洛芬、䒺普生、 三氯生、咖啡因、氯贝酸和 $2,4-\mathrm{D}$ 等七种目标污染物进行 了降解。七种微污染物以 $5 \mu \mathrm{g} \cdot \mathrm{L}^{-1}$ 的浓度投加到 $1000 \mathrm{~L}$ 的反 应器中。结果表明, 三氯生、双氯芬酸和菜普生主要通过 光降解, 去除率在 $99 \%$ 以上。布洛芬和咖啡因被植物吸收, 且去除率与水生藻类植物和细菌活性有关, 其中浮萍和槐 叶藻降解咖啡因效果最好, 去除率大于 $99 \%$; 伊乐藻降解 布洛芬效果最好, 去除率在 $85 \%$ 以上。氯贝酸与 $2,4-\mathrm{D}$ 在水 中的阴离子与植物表面的阴离子产生了斥力, 导致氯贝酸 和 $2,4-\mathrm{D}$ 不能被降解。该实验为我们提供了一个利用水生 植物去除PPCPs的新思路。

\section{5. 存在问题及未来研究趋势}

(1) 到目前为止, 对纯藻降解PPCPS相关研究甚少, 实验一般采用开放式反应器, 实验中大多是藻菌共生体 系, 没有利用纯藻进行实验。在降解过程中菌体的具体工 作原理及纯藻对PPCPs 降解率尚不清楚, 这一问题目前正 在研究当中。

（2）当前采用的微藻和水生藻类植物在降解PPCPS 过程中是否产生变异未可知, 应对处理PPCPs的藻类做生
态毒理学效应分析, 并设计藻类回收处理系统和相应的环 境影响评价体系, 以保证不会对环境生态产生污染。

(3) 可根据藻类处理技术建立实际的污水处理工艺, 例如高效藻类塘。需要构建反应动力学模型用于模拟实际 PPCPs的降解过程, 除了降解PPCPs之外, 藻类对氮磷的吸 收研究可同时开展, 使高效藻类塘既可以去除水中的PPCPS 又可以进一步降解二级处理工艺出水中剩余的氮和磷。

\section{6. 结论}

根据文献研究表明, 藻类降解PPCPs 一般有光降解和 生物降解两种途径。利用藻类降解PPCPs的研究刚刚开始 起步, 对于大多数PPCPs 的降解产物都还处于未知状态, 没有进行深入研究。处理工艺的可行性取决于是否安全地 降解目标化合物, 而在某些情况下, 部分目标污染物会转 化生成毒性更高的化合物或副产物。与传统生物处理工艺 相比较, 利用微藻处理PPCPs的理论还有待进一步研究。 因此, 为了详细综合评价藻类处理工艺, 需对具体的降解 途径机理和形成的产物进行阐明和鉴定。

\section{参考文献}

[1] Richardson S D, Ternes T A. Water Analysis: Emerging Contaminants and Current Issues [J]. Analytical Chemistry. 2005, $77(12)$ : 3807-3838.

[2] Glassmeyer S T, Furlong E T, Kolpin D W, et al. Transport of Chemical and Microbial Compounds from Known Wastewater Discharges: Potential for Use as Indicators of Human Fecal Contamination $[\mathrm{J}]$. Environmental Science \& Technology. 2005, $39(14)$ : $5157-5169$

［3］周海东, 黄霞, 文湘华. 城市污水中有关新型微污染物PPCPs 归趋研究的进展. 环境工程学报. 2007 (12) : 1-9。Haidong Zhou, Xia Huang, Xianghua Wen. 2007 (12) : 1-9。

[4] 王丹, 隋倩, 赵文涛, 等. 中国地表水环境中药物和个人护 理品的研究进展. 科学通报. 2014(09): 743-751. Dan Wang, Qian Sui, Wentao Zhao, 等. 2014(09) :743-751 (in Chinese).

[5] Triebskorn R, Casper H, Heyd A, et al. Toxic effects of the non-steroidal anti-inflammatory drug diclofenac [J]. Aquatic Toxicology. 2004, 68(2) : 151-166.

[6] Abargues M R, Ferrer J, Bouzas A, et al. Removal and fate of endocrine disruptors chemicals under lab-scale postreatment stage. Removal assessment using light, oxygen and microalgae [J]. Bioresource Technology. 2013, 149: 142-148.

[7] Thomas A T, Adriano J. Human Pharmaceuticals, Hormones and Fragrances: The Challenge of Micropollutants in Urban Water Management, [M]. Science of the Total Environment, 2007: 729. 
[8] Stackelberg P E, Furlong E T, Meyer M T, et al. Persistence of pharmaceutical compounds and other organic wastewater contaminants in a conventional drinking-water-treatment plant [J]. Science of The Total Environment. 2004, $329(1-3)$ : 99-113.

[9] Hartig C, Storm T, Jekel M. Detection and identification of sulphonamide drugs in municipal waste water by liquid chromatography coupled with electrospray ionisation tandem mass spectrometry [J]. J Chromatogr A. 1999, 854(1-2) : 163-173.

[10] Hirsch R, Ternes T, Haberer K. Occurrence of antibiotics in the aquatic environment $[\mathrm{J}]$. Science of the Total Environment. 1999, 225(1-2) : 109-118.

[11] Golet E M, Alder A C, Hartmann A, et al. Trace Determination of Fluoroquinolone Antibacterial Agents in Urban Wastewater by Solid-Phase Extraction and Liquid Chromatography with Fluorescence Detection[J]. Analytical Chemistry. 2001, 73(15) : 3632-3638.

[12] Kolpin D W, Furlong E T, Meyer M T, et al. Pharmaceuticals, Hormones, and Other Organic Wastewater Contaminants in U. S. Streams, 1999-2000: A National Reconnaissance [J]. Environmental Science \& Technology. 2002, 36(6): 1202-1211.

[13] Sacher F, Lang F T, Brauch H J, et al. Analytical methods and results of a monitoring program in Baden-Wurttemberg, Germany Pharmaceuticals in groundwaters [J]. 2001, 938(1-2): 199-210.

[14] Golet E M, Alder A C, Giger W. Environmental Exposure and Risk Assessment of Fluoroquinolone Antibacterial Agents in Wastewater and River Water of the Glatt Valley Watershed, Switzerland [J]. Environmental Science \& Technology. 2002, 36(17) : 3645-3651.

[15] Ternes T A, Herrmann N, Bonerz M, et al. A rapid method to measure the solid-water distribution coefficient (Kd) for pharmaceuticals and musk fragrances in sewage sludge [J]. Water Research. 2004, 38(19): $4075-4084$

[16] Carballa M, Omil F, Lema J M. Removal of cosmetic ingredients and pharmaceuticals in sewage primary treatment [J]. Water Research. 2005, 39(19): $4790-4796$.

[17] Carballa M, Omil F, Lema J M, et al. Behavior of pharmaceuticals, cosmetics and hormones in a sewage treatment plant [J]. Water Research. 2004, 38(12) : 2918-2926.

[18] 王艺, 陈川, 张子峰, 等. 甲基硅氧烷在污水处理厂中的 环境行为. 化工学报. 2016(01):83-88. Yi Wang, Chuan Chen, Zifeng Zhang，等. 2016(01)：83-88。

[19] Tratnyek P W E S. Quantitative structure-activity relationships for chemical reductions of organic contaminants [J]. 2003, 22 (8) : 1733-1742.
[20] Clara M, Kreuzinger N, Strenn B, et al. The solids retention time-a suitable design parameter to evaluate the capacity of wastewater treatment plants to remove micropollutants [J]. Water Research. 2005, $39(1)$ : 97-106.

[21] Göbel A, Thomsen A, Mcardell C S, et al. Occurrence and Sorption Behavior of Sulfonamides, Macrolides, and Trimethoprim in Activated Sludge Treatment [J]. Environmental Science \& Technology. 2005, 39 (11): 3981-3989.

[22] Carballa M, Omil F, Ternes T, et al. Fate of pharmaceutical and personal care products (PPCPs) during anaerobic digestion of sewage sludge [J]. Water Research. 2007, 41 (10) : 2139-2150.

[23] Van De Plassche E J, Balk F. Environmental risk assessment of the polycyclic musks AHTN and HHCB according to the EU-TGD [R]., 1997.

[24] Kupper T, Berset J D, Etter-Holzer R, et al. Concentrations and specific loads of polycyclic musks in sewage sludge originating from a monitoring network in Switzerland [J]. Chemosphere. 2004, 54 (8) : $1111-1120$.

[25] Khan S J, Ongerth J E. Estimation of pharmaceutical residues in primary and secondary sewage sludge based on quantities of use and fugacity modeling $[\mathrm{J}]$. Water Science \& Technology. 2002, 46(3): 105-113.

[26] Andersen H, Siegrist H, Halling-Sørensen B, et al. Fate of Estrogens in a Municipal Sewage Treatment Plant [J]. Environmental Science \& Technology. 2003, 37 (18): 4021-4026.

[27] Stamatelatou K, Frouda C, Fountoulakis M, et al. Pharmaceuticals and health care products in wastewater effluents: the example of carbamazepine [J]. Water Science and Technology: Water Supply. 2003, 4(3) : 131-137.

[28] 高颖. 德国污水厂四级处理：微污染有机物质控制技术现 状和展望. 中国给水排水, 2016. Ying Gao. 中国给水排 水, 2016 。

[29] Ternes T A, Stuber J, Herrmann N, et al. Ozonation: a tool for removal of pharmaceuticals, contrast media and musk fragrances from wastewater $[\mathrm{J}]$. Water Res. 2003, $37(8)$ : 1976-1982.

[30] Gunten U V. Ozonation of drinking water: Part I. 0xidation kinetics and product formation[J]. Water Research. 2003, 7 (37): 1443-1467.

[31] Huber M M, Canonica S, Park G Y. Oxidation of Pharmaceuticals During Ozonation and Advanced 0xidation Processes [J]. Environmental Science \& Technology. 2003, 5(37): 1016-1024. 
[32] Esplugas S, Bila D M, Krause L G T, et al. Ozonation and advanced oxidation technologies to remove endocrine disrupting chemicals (EDCs) and pharmaceuticals and personal care products (PPCPs) in water effluents $[\mathrm{J}]$. Journal of Hazardous Materials. 2007, $149(3): 631-642$.

[33] Adams C, Asce M, Wang Y, et al. Removal of Antibiotics from Surface and Distilled Water in Conventional Water Treatment Processes [J]. Journal of Environmental Engineering. 2003, 128(3) : 253-260.

[34] Andreozzi R, Caprio V, Marotta R, et al. Paracetamol oxidation from aqueous solutions by means of ozonation and $\mathrm{H}_{2} \mathrm{O}_{2} / \mathrm{UV}$ system [J]. Water Res. 2003, $37(5)$ : 993-1004.

[35] Andreozzi R, Caprio V, Marotta R, et al. Ozonation and $\mathrm{H}_{2} \mathrm{O}_{2} /$ UV treatment of clofibric acid in water: a kinetic investigation [J]. Journal of Hazardous Materials. 2003, $103(3)$ : 233-246.

[36] Kang Y W, Hwang K. Effects of reaction conditions on the oxidation efficiency in the Fenton process [J]. Water Research. 2000, 34(10): 2786-2790.

[37]周雪飞, 陈家斌, 张亚雷. 膜处理和活性炭吸附去除水环 境中的 PPCPs. 给水排水. 2009 (S2) : 81-84. Xuefei Zhou, Jiabin Chen, Yalei Zhang. 2009 (S2) : 81-84。

[38] Snyder S A, Adham S, Redding A M, et al. Role of membranes and activated carbon in the removal of endocrine disruptors and pharmaceuticals [J]. Desalination. 2007, $202(1-3)$ : 156-181.

[39] Radjenović J, Petrović M, Ventura F, et al. Rejection of pharmaceuticals in nanofiltration and reverse osmosis membrane drinking water treatment $[\mathrm{J}]$. Water Research. 2008, 42(14): 3601-3610.

[40] Boelee N C, Temmink H, Janssen M, et al. Nitrogen and phosphorus removal from municipal wastewater effluent using microalgal biofilms $[\mathrm{J}]$. Water Research. 2011, 45(18): 5925-5933.

[41] Roeselers G, Loosdrecht M C M V, Muyzer G. Phototrophic biofilms and their potential applications [J]. Journal of Applied Phycology. 2008, 20 (3) : 227-235.

[42] Pittman J K, Dean A P, Osundeko 0. The potential of sustainable algal biofuel production using wastewater resources [J]. Bioresource Technology. 2011, $102(1): 17-25$.

[43] Cameron E, Mairet F, Bernard 0, et al. Anaerobic Digestion of Microalgae: Identification for
Optimization and Control [J]. IFAC Proceedings Volumes. 2011, 44(1) : 5032-5027.

[44] Mehta C M, Khunjar W 0, Nguyen V, et al. Technologies to Recover Nutrients from Waste Streams: A Critical Review [J]. Critical Reviews in Environmental Science and Technology. 2014, 45(4): 385-427.

[45] Harrass M C, Kindig F A C. Responses of blue-green and green algae to streptomycin in unialgal and paired culture [J]. Aquatic Toxicology. 1985, 6: 1-11.

[46] Wilson B A, Smith V H, Denoyelles F, et al. Effects of Three Pharmaceutical and Personal Care Products on Natural Freshwater Algal Assemblages [J]. Environmental Science \& Technology. 2003, $37(9)$ : $1713-1719$

[47] Delorenzo M E, Fleming J. Individual and Mixture Effects of Selected Pharmaceuticals and Personal Care Products on the Marine Phytoplankton Species Dunaliella tertiolecta [J]. Archives of Environmental Contamination and Toxicology. 2008, $54(2)$ : 203-210.

[48] Matamoros V, Uggetti E, García J, et al. Assessment of the mechanisms involved in the removal of emerging contaminants by microalgae from wastewater: a laboratory scale study $[\mathrm{J}]$. Journal of Hazardous Materials. 2016, 301: 197-205.

[49] de Wilt A, Butkovskyi A, Tuantet K, et al. Micropollutant removal in an algal treatment system fed with source separated wastewater streams $[\mathrm{J}]$. Journal of Hazardous Materials. 2016, 304: 84-92.

[50] de Godos I, Munoz R, Guieysse B. Tetracycline removal during wastewater treatment in high-rate algal ponds [J]. J Hazard Mater. 2012, 229-230: 446-449.

[51] Matamoros V, Uggetti E, García J, et al. Assessment of the mechanisms involved in the removal of emerging contaminants by microalgae from wastewater: a laboratory scale study [J]. Journal of Hazardous Materials. 2016, 301: 197-205.

[52] Daughton C G. Pharmaceuticals and Personal Care Products in the Environment: Agents of Subtle Change [J]. Environmental Health Perspectives. 1999, 107 : 907-938.

[53] Matamoros V, Nguyen L X, Arias C A, et al. Evaluation of aquatic plants for removing polar microcontaminants: A microcosm experiment [J]. Chemosphere. 2012, 88(10): 1257-1264. 